\title{
Psychosocial aspects of children and families of children treated with automated peritoneal dialysis
}

\author{
Katarzyna Kiliś-Pstrusińska • Anna Wasilewska • Anna Medyńska • \\ Irena Bałasz-Chmielewska • Ryszard Grenda • Agnieszka Kluska-Jóźwiak • \\ Beata Leszczyńska • Ilona Olszak-Szot • Monika Miklaszewska • Maria Szczepańska • \\ Marcin Tkaczyk • Agnieszka Urzykowska • Katarzyna Zachwieja • Maria Zajączkowska • \\ Helena Ziółkowska • Ilona Zagożdżon • Danuta Zwolińska
}

Received: 28 January 2013 / Revised: 21 May 2013 / Accepted: 31 May 2013 / Published online: 16 August 2013

(C) The Author(s) 2013. This article is published with open access at Springerlink.com

\begin{abstract}
Background The aim of this study was to analyze psychosocial aspects of chronic kidney disease (CKD) in children treated with automated peritoneal dialysis (APD).

Methods The study assessed 41 children $>2$ (range 2.1-18) years of age and their parents. Data concerning the illness and sociodemographic parameters were collected. Patients completed the Paediatric Quality of Life Inventory (PedsQL) and their parents the PedsQL-proxy version, General Health Questionnaire (GHQ-12), Berlin Social Support Scales (BSSS), and Caregiver's Burden Scale (CBS).

Results Parents rated their children's overall health-related quality of life (QoL) as well as their physical and emotional
\end{abstract}

K. Kiliś-Pstrusińska $(\bowtie) \cdot$ A. Medyńska $\cdot$ D. Zwolińska Department of Paediatric Nephrology, Wrocław Medical University, ul. Borowska 213, 50-556 Wrocław, Poland

e-mail: katarzyna.kilis-pstrusinska@am.wroc.pl

A. Wasilewska

Department of Paediatrics and Nephrology,

Medical University of Białystok, Białystok, Poland

I. Bałasz-Chmielewska • I. Zagożdżon

Department of Paediatric \& Adolescent Nephrology \&

Hypertension, Medical University of Gdansk, Gdansk, Poland

R. Grenda $\cdot$ A. Urzykowska

Department of Nephrology, Kidney Transplantation and

Hypertension, Children's Memorial Health Institute,

Warsaw, Poland

A. Kluska-Jóźwiak

Department of Paediatric Cardiology and Nephrology,

Poznan University of Medical Sciences, Poznan, Poland

B. Leszczyńska $\cdot$ H. Ziółkowska

Department of Paediatrics and Nephrology,

Medical University of Warsaw, Warsaw, Poland functioning lower than the patients themselves. The majority of primary caregivers had a medium level of the total burden index in the CBS and higher values in the scales need for support and perceived available support than in the received support (BSSS). In the GHQ-12, $51.2 \%$ of primary caregivers had scores $>2$ points, which indicated the possible occurrence of abnormal mental functioning.

Conclusions Financial support for patients' families is necessary. Parents who provide primary care to children on PD require, above all, emotional support and assistance in selffulfilment. More than half of them may have impaired mental function. There is the strong need to provide continuous psychological care for caregivers. Differences in perception

\section{Olszak-Szot}

Department of Nephrology, Children Hospital, Toruń, Poland

M. Miklaszewska $\cdot$ K. Zachwieja

Polish-American Children's Hospital, Jagiellonian University, Krakow, Poland

M. Szczepańska

Clinic of Paediatrics, Nephrology and Endocrinology,

Silesian Medical University, Zabrze, Poland

M. Tkaczyk

Nephrology Division, Polish Mothers' Memorial Hospital

Research Institute, Łodź, Poland

M. Zajaczkowska

Department of Paediatric Nephrology, Medical University of Lublin, Lublin, Poland 
of the children's activity in varied areas by the patients themselves and their caregivers may contribute to further problems within families.

Keywords Children $\cdot$ Peritoneal dialysis $\cdot$ Health-related quality of life $\cdot$ Parents' perceived burdens $\cdot$ Primary caregivers

\section{Introduction}

Home peritoneal dialysis (PD) is the preferred chronic dialysis modality for children with end-stage renal disease (ESRD) [1] . Thanks to technological advances, inter alia, various PD regimens and solutions, as well as clinical experience, patient survival and somatic condition has improved $[1,2]$. Nevertheless, impaired health-related quality of life (HRQoL) among patients on dialysis remains a challenge [3]. Patient's QoL is closely related to the family, especially when the patient is on PD. In addition to the typical parental responsibilities and activities of providing support, parents must also be engaged in the therapeutic process $[4,5]$. In many cases, the home environment is medicalized, with family members becoming the main caregivers burdened with many responsibilities related to PD and thus affects the life of the patient's family. On the other hand, each parent's perception of their psychosocial situation, as well as their reaction to it, may affect the relationship between parents and their children and the child's functioning $[6,7]$.

Before the decision concerning renal replacement treatment (RRT) is made, it is necessary to assess the family's social, psychological, and economic background and recognize the needs of parents/caregivers $[2,8]$. Disregarding those factors creates the risk of complicating the method. Thus far, psychosocial aspects of children with chronic kidney disease (CKD) treated with automated peritoneal dialysis (APD) have rarely been analyzed, and never in Poland. Therefore, we conducted a multicenter national study with the aim of analyzing the psychosocial situation in families of children treated with APD. We gave particular attention to parents' perceived burdens. We considered children's HRQoL as perceived by themselves and as by their parents, because perceiving the QoL of sick children is one element of assessing the family situation. Another aim was to determine in which areas of everyday life the families of children treated with APD require support.

\section{Methods}

Eleven of 12 pediatric dialysis centers in Poland participated in the cross-sectional national study. The research was conducted on children with ESRD treated with APD and their parent-proxies between September and December 2011. The study protocol adhered to the Declaration of Helsinki and was approved by the Ethics Committee of Wrocław Medical University. Written informed consent was obtained from all participants $>16$ years of age and all parents before enrollment in the study. Verbal consent was obtained from patients $<16$ years where possible. Inclusion criteria for children were as follows:

1. CKD diagnosed at least 12 months prior to the study,

2. PD beginning at least 3 months prior to the study,

3. age $\geq 2$ years, and

4. informed consent.

Exclusion criteria for children comprised:

1. a history of severe to profound mental retardation,

2. renal, other solid-organ, bone marrow, or stem cell transplantation,

3. cancer/leukemia diagnosis,

4. hospitalization within 14 days (excluding hospitalization due to peritoneal dialysis control visit), and

5. a significant life event unrelated to their kidney disease in the past 30 days, such as losing a family member.

In Poland, there is a common treatment protocol for managing children on APD, with PD nurses who meet the families predialysis. The nurses work in a dialysis center. Home visits prior to dialysis are done by district nurses who also do the follow-up visits. The district nurses are in constant contact with the PD nurses. The PD family has access to district social workers and psychologists.

Medical files were analyzed to obtain the following data: primary diagnosis of kidney disease, patient's age at time of CKD diagnosis, illness duration, APD duration, nephrologic care duration, additional nonrenal comorbidities, place of living and distance from a nephrologic center, and family history. Additionally, school-age children and their parents were asked about schooling level and special education requirements (supplementary tutoring or an individualized education program). Then, both parents were invited to fill out questionnaires regarding changes in their families after the child's CKD diagnosis and their relationship with the people in their immediate surroundings. Questionnaires were completed on a visit to the renal center, and each parent filled out the questionnaire separately.

In the next step, we used Pediatric Quality of Life Inventory (PedsQL) 4.0 Generic Core Scales [9, 10]. We obtained the user agreement from Christelle Berne, Mapy Research Institute in Lyon, France. In addition, parents underwent the following test studies: the 12-item General Health Questionnaire (GHQ-12) [11], Berlin Social Support Scales (BSSS) [12, 13], and the Caregiver's Burden Scale (CBS) [14]. All tests were translated and validated in Poland [15-17]. Cronbach's $\alpha$ for GHQ-12 is 0.72 and for the four subscales of BSSS ranges from 0.70 to 
0.86. Cronbach's $\alpha$ is 0.88 for the total scale of CBS and ranges from 0.74 to 0.82 for the subscales.

The PedsQL assesses physical, emotional, social, and school functioning in children and adolescents. The measure comprises a report from children 5-18 years of age and a parent report for children between 2 and 18 years of age regarding the child's HRQoL. The PedsQL 4.0 Generic Core Scales were specifically designed to measure the core health dimensions outlined by the World Health Organization.

The GHQ-12 measures psychological distress and is used to detect nonpsychotic psychiatric disorders such as depression or anxiety in adults [11]. The scale asks whether the respondent has experienced a particular symptom or behavior recently. Each item is rated on a four-point scale (less than usual, no more than usual, rather more than usual, or much more than usual). The GHQ-12 is brief, easy to complete, and its application in research settings as a screening tool is well documented. We used the original scoring method in our study (response categories score: $0,0,1$, and 1 , respectively). This produces scores ranging from 0 to 12 ; the higher values indicate more psychological symptoms.

The original BSSS includes six independent subscales (perceived available support, need for support, support seeking, actual received support, provided support, and protective buffering) and measures both the cognitive and behavioral aspects of social support $[12,13]$. With the authors' consent, four subscales were used for this study: perceived available support ( 8 items), need for support (4 items), support seeking ( 5 items), and actual received support (15 items). The perceived support subscale comprises items of emotional and instrumental support; the overall received support includes items of emotional, instrumental, and informational support. The response format is the same for all subscales. Individuals rate their agreement with the statements on a four-point scale [strongly disagree (1), somewhat disagree (2), somewhat agree (3), and strongly agree (4)]. An average mean within the range of 1-4 was calculated for each subscale. A higher score indicated greater burden.

The CBS uses 22 items to assess the subjective burden of caregivers to chronically ill individuals [14]. All items are scored between 1 and 4 (not at all, seldom, sometimes, often) and cover areas such as the caregiver's health, feeling of psychological well-being, relationships, social network, physical workload, and environmental aspects that might prove important. The scale was divided into five indices: general strain (8 items), socialization (3 items), disappointment (5 items), emotional involvement (3 items), and environment ( 3 items). The total burden index is the mean of all 22 items. The higher the score, the greater the burden. The overall mean of a caregiver's burden score was defined as low burden (1.00-1.99), medium burden (2.00-2.99), and high burden (3.00-4.00) [14].
All tests were designed according to the general protocol and administration guidelines.

Statistical analyses

Statistical analyses were performed using $\mathrm{R}$ for Windows, version 2.15.1 (The R Foundation for Statistical Computing, Vienna, Austria) and MedCalc for Windows, version 12.3.1.0 (MedCalc Software, Mariakerke, Belgium). Medians, quartiles, frequencies, and percentages were reported to describe data, as appropriate. Qualitative analyses were conducted using a chi-squared test or Fisher's exact test. Quantitative variables were tested for normality distribution by a Kolmogorov-Smirnov test. For comparison between groups, the Mann-Whitney and Kruskal-Wallis tests were used, as appropriate. Spearman's correlation analysis was used to determine the correlation between parameters. A $p$ value $<0.05$ was considered statistically significant.

\section{Results}

Taking into consideration inclusion and exclusion criteria, except for parents' consent, 50 children were qualified for inclusion in the study/examination. Final studies were conducted among 41 children with APD whose parents gave their consent. Among the 82 parents and/or their guardians (all Polish), data from 40 mothers (one mother maintains no contact with her sick child) and 39 fathers were analyzed. Four fathers do not live with their families, but two mothers provided the fathers' information. In subsequent parts of the analysis, only the results of fully completed surveys were taken into consideration.

\section{Survey results}

Characteristics of the examined children are presented in Table 1. All children at their compulsory school age ( $>5$ years, $n=30$ ) carried on their education. Thirteen children (44.8\%) went to school (including two attending special schools); 16 patients were home-schooled (55.2\%). One child did not provide information with regard to the type of learning. Eleven pupils (37.93\%) required no help with their studying; the remaining 18 children $(62.07 \%)$ received assistance from their parents. The number of school days (or lesson days at home) missed in the previous 6 months was (median, quartiles) $10(0-30)$.

The characteristics of APD children's families, their parents in particular, are presented in Table 2. Most children were growing up in complete families; only five of them lived with one parent (four with the mother and one with the father). Thirty children had siblings (26 of them one or two; four had three to six). Family household incomes come from 
Table 1 Basic characteristics of automated peritoneal dialysis (APD) children
$P D$ peritoneal dialysis; $C K D$ chronic kidney disease

${ }^{\mathrm{a}}$ In the previous 6 months

\begin{tabular}{|c|c|c|}
\hline Parameter & Number $=41$ & Percentage \\
\hline Age, mean $\pm \mathrm{SD}$, (years) & $9.24 \pm 5.09$ & \\
\hline Children $<5$ & 10 & 24.4 \\
\hline$\geq 5$ & 31 & 75.6 \\
\hline \multicolumn{3}{|l|}{ Gender } \\
\hline Female & 14 & 34.15 \\
\hline Male & 27 & 65.85 \\
\hline \multicolumn{3}{|l|}{ Cause of CKD } \\
\hline Chronic glomerulonephritis & 13 & 31.71 \\
\hline Anomaly of kidney and urinary tract and chronic pyelonephritis & 15 & 35.58 \\
\hline Hereditary kidney disease & 9 & 21.95 \\
\hline Others & 3 & 7.32 \\
\hline Unknown cause & 1 & 2.44 \\
\hline \multicolumn{3}{|l|}{ Comorbidity } \\
\hline Yes & 6 & 14.6 \\
\hline No & 35 & 85.4 \\
\hline \multicolumn{3}{|l|}{ Family renal history } \\
\hline Yes & 4 & 9.76 \\
\hline No & 37 & 90.14 \\
\hline \multicolumn{3}{|l|}{ Place of residence (size) } \\
\hline Village or town $<50,000$ residents & 27 & 65.85 \\
\hline Town $50,000-100,000$ residents & 3 & 7.32 \\
\hline Town $>100,000$ residents & 11 & 26.83 \\
\hline Age (years) at CKD diagnosis, median (quartiles), & $1.5(0.02-6.0)$ & \\
\hline CKD duration (years), median (quartiles) & $4.17(2.21-8.15)$ & \\
\hline Nephrological care duration (years), median (quartiles) & $4.0(2.25-8.18)$ & \\
\hline PD therapy (years), median (quartiles) & $1.98(0.71-2.25)$ & \\
\hline Distance from nephrology center $(\mathrm{km})$, median (quartiles) & $65.0(21.5-102.5)$ & \\
\hline Number of hospitalizations ${ }^{\mathrm{a}}$, median (quartiles) & $6.0(3.0-6.0)$ & \\
\hline
\end{tabular}

various sources but mostly from professional careers, indicated by $82.5 \%$ of parents (multiple choice options). Other sources of income included annuity and/or pension, which were indicated by $15 \%$ of parents, and social welfare in $47 \%$ of respondents. Parents' perceived change in their families' situation after the child's CKD diagnosis is summarized in Table 3. No statistically significant differences were found with respect to the parents' gender.

Use of social welfare and unemployment, and deterioration in financial situation in parents' evaluation (Tables 2 and 3) showed a harsh economic situation for the family.

Each parent was asked to evaluate their relationships with the people in their immediate surroundings and with medical staff by assigning points from 1 (very bad) to 5 (very good). Results are shown in Table 4. Differences between fathers' and mothers' assessments were not statistically significant. In each family, parents indicated the sick child's main caregiver. The group of primary caregivers comprised 40 mothers and one father. Guardians (henceforth referred to as primary caregivers) were asked to share their expectations toward medical personnel. Twelve individuals (29.3\%) described their expectations toward nurses. In addition to general terms, such as "providing care" and "help", attention focused on the assistance provided during the hospital stay, sharing more information regarding $\mathrm{PD}$, greater professionalism, and better contact with the child. With respect to physicians, nine individuals (22\%) expected "good care" and more detailed information about the patient, mainly about the projected course of the disease and their child's future. Only 15 primary caregivers $(36.59 \%)$ asserted that their child's kidney transplantation was discussed.

Test results

The PedsQL test results in the entire group of parents who provide primary care for APD children $(n=41)$ were significantly lower in relation to results among parents of healthy children published in the literature, both within the scope of overall HRQoL $(51.0 \pm 16.42$ vs $81.34 \pm 15.92, p<0.01)$ and 
Table 2 Basic characteristics of parents and families of children on automated peritoneal dialysis

\begin{tabular}{|c|c|c|}
\hline Parameter & Number & Percentage \\
\hline Mothers & $40^{\mathrm{a}}$ & 100 \\
\hline Age, mean $\pm \mathrm{SD}$, (years) & $28.16 \pm 6.0$ & \\
\hline \multicolumn{3}{|l|}{ Mother's education level } \\
\hline Elementary/trade school & 14 & 35 \\
\hline High school & 15 & 37.5 \\
\hline University degree & 11 & 27.5 \\
\hline \multicolumn{3}{|l|}{ Employment } \\
\hline Yes & 29 & 72.5 \\
\hline No & 11 & 27.5 \\
\hline \multicolumn{3}{|l|}{ Healthy } \\
\hline Yes & 34 & 85 \\
\hline No & 6 & 15 \\
\hline Fathers & $39^{\mathrm{b}}$ & 100 \\
\hline Age, mean $\pm \mathrm{SD}$, (years) & $40.03 \pm 9.27$ & \\
\hline \multicolumn{3}{|l|}{ Father's education level } \\
\hline Elementary/trade school & 24 & 61.54 \\
\hline High school & 7 & 17.95 \\
\hline University degree & 8 & 20.51 \\
\hline \multicolumn{3}{|l|}{ Employment ${ }^{\mathrm{c}}$} \\
\hline Yes & 30 & 81.1 \\
\hline No & 7 & 18.9 \\
\hline \multicolumn{3}{|l|}{ Healthy } \\
\hline Yes & 33 & 84.62 \\
\hline No & 6 & 15.38 \\
\hline \multicolumn{3}{|l|}{ Family } \\
\hline Full & 36 & 87.8 \\
\hline Single-parent & 5 & 12.2 \\
\hline \multicolumn{3}{|l|}{ Sibling } \\
\hline Yes & 30 & 73.17 \\
\hline No & 11 & 26.83 \\
\hline
\end{tabular}

$S D$ standard deviation

${ }^{a}$ One mother does not live with her child (no contact). ${ }^{\mathrm{b}}$ Four fathers do not live with their families, but in the case of two fathers, the mothers provided their information. ${ }^{\mathrm{c}}$ No information was provided for two fathers

in individual subscales: physical functioning $(49.24 \pm 18.75$ vs $83.26 \pm 19.98, p<0.01)$, emotional functioning $(55.12 \pm 18.5 \mathrm{vs}$ $80.28 \pm 16.99, p<0.01)$, social functioning $(55.24 \pm 22.11 \mathrm{vs}$ $82.15 \pm 20.08, p<0.01)$, and school functioning $(55.7 \pm 28.93$ vs $76.91 \pm 20.08, p<0.01)$ [9]. Similarly, PedsQL test results among APD patients (5-18 years of age) were significantly lower statistically $(p<0.01)$ than in a group of healthy children: physical functioning $(61.19 \pm 18.57$ vs $86.86 \pm 13.88)$, emotional functioning ( $66.29 \pm 14.08$ vs $78.21 \pm 18.64)$, social functioning $(69.19 \pm 22.11$ vs $84.04 \pm 17.43)$, school functioning $(65.83 \pm 28.93$ vs $79.92 \pm 16.93)$, and overall HRQoL $(65.83 \pm 28.93$ vs $82.87 \pm 13.6)$ [9]. Table 5 summarizes the
PedsQL test results among children between 5 and 18 years of age $(n=31)$ and both parents. All primary guardians and 13 "secondary parents" completed the tests. Both parents rated their children's physical and emotional functioning as well as their overall HRQoL as significantly lower than the sick children did themselves. The patients rated their social functioning higher than did their primary caregivers. No significant differences were found in the evaluation of school functioning between the studied groups. The intraclass correlation coefficient (ICC) between primary parent and child reports was calculated to be 0.74 . The ICC between secondary parent and child reports and between primary and secondary parent reports were calculated to be 0.68 and 0.64 , respectively.

Parents' test results are demonstrated in Table 6. There were no significant differences within the scope of social support (perceived available support, the need for such support, support seeking, and received support) between primary and secondary parents (BSSS results). The CB scale demonstrated that the average total burden index in primary caregivers was medium. Results obtained also correspond with medium burden in the following subscales: general strain, social isolation, and disappointment. Low levels of burden were observed in the emotional involvement and environment subscales. The results in the secondary parents group indicate lower general strain than in primary guardians; no significant differences were demonstrated with respect to the remaining subscales; however, the average total burden index (2.0) borders on low and medium burden.

GHQ-12 results in the group of primary caregivers were significantly higher than in the group of secondary caregivers. In addition, scores $>2$ points, which indicated the possible occurrence of abnormal mental functioning, were observed in $51.2 \%$ of primary parents and in only $23.53 \%$ of parents who did not provide primary care. There was no significant correlation between BSSS, CBS, and GHQ-12 and parents' age, education, professional career, family structure (complete/single parent), having or not having other children, and source of income (professional career vs welfare benefits). The level of general strain (CBS) declared by women was significantly higher than that declared by men $(2.63,1.91-3.0$ vs $1.94,1.75-2.75, p=0.03)$. Guardians who live in medium-sized cities demonstrated a higher level of social isolation $(3,2.33-3.42)$ and total burden index $(2.59,2.0-2.77)$ than residents in rural areas and in large cities (social isolation 2.0, 1.33-2.75 and 2.33, 1.37-2.84, respectively; $p=0.02$; total burden index: $1.82,1.58-2.48$ and $2.16,1.7-2.73$, respectively; $p=0.01$ ). No significant differences were found between residents in rural areas and large cities in this regard.

Perceived available instrumental support was lowest among small-town residents $(3.25,2.19-4.0)$ in comparison with village residents $(3.75,3.75-4)$ and city residents $(4.0$, 
Table 3 Changes in the family observed by the parents after chronic kidney disease (CKD) diagnosis in the child

\begin{tabular}{|c|c|c|c|c|c|c|c|c|c|}
\hline & \multicolumn{4}{|c|}{ Mothers' evaluation $n=40^{\mathrm{a}}$} & \multicolumn{4}{|c|}{ Fathers' evaluation $n=35^{\mathrm{a}}$} & \multirow[t]{3}{*}{$P$ value ${ }^{\mathrm{d}}$} \\
\hline & \multicolumn{2}{|l|}{ Yes } & \multicolumn{2}{|l|}{ No } & \multicolumn{2}{|c|}{ Yes } & \multicolumn{2}{|l|}{ No } & \\
\hline & $n$ & $\%$ & $n$ & $\%$ & $n$ & $\%$ & $n$ & $\%$ & \\
\hline Change of location of residence & 3 & 7.5 & 37 & 92.5 & 1 & 2.86 & 34 & 97.14 & 0.61 \\
\hline Changes made to current residence & 24 & 60 & 16 & 40 & 20 & 57.14 & 15 & 42.86 & 0.81 \\
\hline $\begin{array}{l}\text { Change in financial status } \\
\text { Deterioration }\end{array}$ & $\begin{array}{l}16 \\
16\end{array}$ & $\begin{array}{l}40 \\
40\end{array}$ & 24 & 60 & $\begin{array}{l}16 \\
16\end{array}$ & $\begin{array}{l}45.71 \\
45.71\end{array}$ & 19 & 54.29 & 0.64 \\
\hline Improvement & 0 & & & & 0 & & & & \\
\hline Change of income source & 9 & 22.5 & 31 & 77.5 & 7 & 20 & 28 & 80 & 1 \\
\hline Caring for the child & & & & & & & & & 0.14 \\
\hline $\begin{array}{l}\text { Mother } \\
\text { Father }\end{array}$ & $\begin{array}{l}34 \\
1\end{array}$ & $\begin{array}{l}85 \\
2.5\end{array}$ & & & $\begin{array}{l}24 \\
1\end{array}$ & $\begin{array}{l}68.57 \\
2.86\end{array}$ & & & \\
\hline Both parents & 5 & 12.5 & & & 10 & 28.57 & & & \\
\hline $\begin{array}{l}\text { Participation of other people in child care } \\
\text { Grandparents }\end{array}$ & $\begin{array}{l}22 \\
18\end{array}$ & 55 & 18 & 45 & $\begin{array}{l}18 \\
15\end{array}$ & 51.34 & 17 & 48.57 & 0.81 \\
\hline Siblings & 2 & & & & 3 & & & & \\
\hline Cousins & 2 & & & & 0 & & & & \\
\hline $\begin{array}{l}\text { Changes in attitude toward the ill child } \\
\text { Improvement }\end{array}$ & $\begin{array}{l}15 \\
14\end{array}$ & $\begin{array}{l}37.5 \\
35.0\end{array}$ & 25 & 62.5 & $\begin{array}{l}9 \\
9\end{array}$ & $\begin{array}{l}25.71 \\
25.71\end{array}$ & 26 & 74.29 & 0.32 \\
\hline Deterioration & 1 & 2.5 & & & 0 & & & & \\
\hline $\begin{array}{l}\text { Changes in attitude toward other children } \\
\text { Improvement }\end{array}$ & $\begin{array}{l}\mathrm{b}_{4} \\
4\end{array}$ & $\begin{array}{l}13.33 \\
13.33\end{array}$ & 26 & 86.67 & $\begin{array}{l}{ }^{\mathrm{c}} 4 \\
3\end{array}$ & $\begin{array}{l}16.0 \\
12\end{array}$ & 21 & 84.0 & 1 \\
\hline Deterioration & 0 & & & & 1 & 4 & & & \\
\hline $\begin{array}{l}\text { Changes in relations among children } \\
\text { Improvement }\end{array}$ & $\begin{array}{l}{ }^{b} 10 \\
9\end{array}$ & $\begin{array}{l}33.33 \\
30.0\end{array}$ & 20 & 66.67 & $\begin{array}{l}{ }_{6}^{\mathrm{c}} 6 \\
6\end{array}$ & $\begin{array}{l}24 \\
24\end{array}$ & 19 & 76 & 0.55 \\
\hline Deterioration & 1 & 3.33 & & & 0 & & & & \\
\hline $\begin{array}{l}\text { Changes in social contacts } \\
\text { Improvement }\end{array}$ & $\begin{array}{l}25 \\
1\end{array}$ & $\begin{array}{l}62.5 \\
2.5\end{array}$ & 15 & 37.5 & $\begin{array}{l}17 \\
0\end{array}$ & 48.57 & 18 & 51.43 & 0.25 \\
\hline Deterioration & 24 & 60.0 & & & 17 & 48.57 & & & \\
\hline New friendship & 28 & 70 & 12 & 30 & 17 & 48.57 & 18 & 51.43 & 0.09 \\
\hline
\end{tabular}

Data shown as a number of responses (percent)

${ }^{a}$ Data collected from 75 surveys (40 mothers, 35 fathers); missing surveys (7; $7.32 \%$ ): 4 fathers, 1 mother, who do not live in the same household; 2 fathers did not fill out this part of the survey

${ }^{\mathrm{b}} n=30,{ }^{\mathrm{c}} n=25 ;{ }^{\mathrm{d}}$ differences between mothers' and fathers' groups, $p$ value

Table 4 Evaluation of parents' relationships with other people: 1 (very bad), 5 (very good)

\begin{tabular}{|c|c|c|c|c|c|c|c|c|c|c|c|c|}
\hline & \multicolumn{6}{|c|}{ Mother } & \multicolumn{6}{|c|}{ Father } \\
\hline & $n$ & 1 & 2 & 3 & 4 & 5 & $n$ & 1 & 2 & 3 & 4 & 5 \\
\hline Father/mother of the child & 38 & $3(7.9)$ & $1(2.6)$ & $5(13.2)$ & $14(36.8)$ & $15(39.5)$ & 35 & $2(5.7)$ & $1(2.9)$ & $2(5.7)$ & $17(48.6)$ & $13(37.1)$ \\
\hline Child with CKD & 40 & 0 & 0 & $1(2.5)$ & $10(25)$ & $29(72.5)$ & 35 & 0 & 0 & $3(8.6)$ & $14(40)$ & $18(51.4)$ \\
\hline Other children & 30 & 0 & $1(3.3)$ & 0 & $11(36.7)$ & $18(60)$ & 25 & 0 & 0 & $2(8)$ & $13(52)$ & $10(40)$ \\
\hline Friends & 40 & $3(7.5)$ & $3(7.5)$ & $10(25)$ & $12(30)$ & $12(30)$ & 35 & 0 & 0 & $8(22.9)$ & $18(51.4)$ & $9(25.7)$ \\
\hline Nurses & 40 & $1(2.5)$ & 0 & 0 & $15(37.5)$ & $24(60)$ & 25 & $1(4)$ & 0 & $1(4)$ & $13(52)$ & $10(40)$ \\
\hline Doctors & 40 & $1(2.5)$ & 0 & $1(2.5)$ & $13(32.5)$ & $25(62.5)$ & 25 & $1(4)$ & 0 & $2(8)$ & $12(48)$ & $10(40)$ \\
\hline Social workers ${ }^{\mathrm{a}}$ & $40^{\mathrm{a}}$ & $3(7.5)$ & 0 & $1(2.5)$ & $8(20)$ & $11(27.5)$ & $25^{\mathrm{b}}$ & $3(12)$ & $2(8)$ & 0 & $3(12)$ & $3(12)$ \\
\hline
\end{tabular}

CKD chronic kidney disease

Data shown as a number $(\%)$

${ }^{\mathrm{a}} 17$ mothers $(42.5 \%)$ did not deal with social workers; ${ }^{\mathrm{b}} 14$ fathers $(56 \%)$ did not deal with social workers 
Table 5 Paediatric Quality of Life Inventory (PedsQL) 4.0 generic core scales from child's self-report (for ages $5-18 ; n=31$ ) and parent-proxy report (main caregivers and second parents)

\begin{tabular}{lccc}
\hline Characteristic & APD children $n=31$ & Main caregivers of APD children $n=31$ & Second parents of APD children $n=13$ \\
\hline Physical functioning & $62.5(50.0-75.0)^{\mathrm{a}, \mathrm{b}}$ & $56.24(40.63-62.5)$ & $53.13(39.84-65.63)$ \\
Emotional functioning & $70.0(56.25-73.75)^{\mathrm{a}, \mathrm{b}}$ & $55.0(40.0-70.0)$ & $55.0(50.0-71.25)$ \\
Social functioning & $70.0(50.0-90.0)^{\mathrm{a}}$ & $55.0(40.0-70.0)$ & $65.0(53.75-81.25)$ \\
School functioning & $62.5(50.0-75.0)$ & $55.0(35.0-65.0)$ & $60.0(47.5-70.0)$ \\
Overall HRQoL & $64.13(51.09-75.0)^{\mathrm{a}, \mathrm{b}}$ & $54.35(38.87-64.13)$ & $54.35(48.91-63.32)$
\end{tabular}

Data are presented as median and quartiles (first-third quartile)

$A P D$ automated peritoneal dialysis

${ }^{a}$ APD children vs. main caregivers, $p<0.05$

${ }^{\mathrm{b}}$ APD children vs. second parents, $p<0.05$

$3.25-4.0)(p=0.03)$. Parents living in small towns also perceived less available emotional support and sought it to a lesser extent than did others; however, differences were not statistically significant. There was no correlation between BSSS, CBS, and GHQ-12 results and duration of CKD, remaining under the care of a nephrology specialist and the duration of renal replacement therapy, or distance between place of residence and a nephrology center. The method of teaching a child (general; home school) had no effect on parents' test results. Parents who helped their children with their studying differed significantly from parents who did not help their children in terms of currently received information support
$(2.9,2.4-3.2$ vs $3.4,3.0-3.6 ; p=0.004)$ and instrumental support (3.2, 2.4 -3.8 vs $4.0,3.2-4.0 ; p=0.02)$, which they rated lower.

A statistically significant negative correlation was found between PedsQL (total score) and CBS (total burden index) $(r=-0.51, p=0.001)$ and GHQ-12 $(r=-0.55, p=0.0002)$; a positive correlation was found between PedsQL (total score) and certain BSSS subscales: need for support $(r=0.31$, $p=0.05)$, support seeking $(r=0.36, p=0.02)$, perceived available emotional support $(r=0.65, p=0.001)$, and instrumental support $(r=0.32, p=0.04)$. Also, the following correlations were observed: a positive correlation between CBS (total burden index) and GHQ-12 $(r=0.73, p=0.001)$ and negative
Table 6 General Health Questionnaire (GHQ-12), Berlin Social Support Scales (BSSS), and Caregiver's Burden Scale (CBS) test results among parents of children on peritoneal dialysis

Data are presented as median and quartiles (first-third quartile)

${ }^{a}$ Main caregivers vs. second parents, $p$ value

\begin{tabular}{|c|c|c|c|}
\hline & \multicolumn{3}{|l|}{ Parents } \\
\hline & Main caregivers $n=41$ & Second parents $n=17$ & $P$ value $^{\mathrm{a}}$ \\
\hline \multicolumn{4}{|l|}{ Berlin Social Support Scale } \\
\hline \multicolumn{4}{|l|}{ Perceived available support: } \\
\hline Emotional & $3.5(3.0-4.0)$ & $3.25(2.69-3.81)$ & 0.23 \\
\hline Instrumental & $3.75(3.19-4.0)$ & $3.75(2.69-4.0)$ & 0.8 \\
\hline Need for support & $3.25(2.75-3.5)$ & $3.0(2.25-3.31)$ & 0.17 \\
\hline Support seeking & $3.2(2.55-3.8)$ & $2.8(2.2-3.1)$ & 0.14 \\
\hline \multicolumn{4}{|l|}{ Actually received support: } \\
\hline Emotional & $2.8(2.2-2.8)$ & $2.8(2.3-2.8)$ & 0.37 \\
\hline Informational & $3.0(2.6-3.4)$ & $3.0(2.3-3.4)$ & 0.48 \\
\hline Instrumental & $3.4(2.6-4.0)$ & $3.2(2.3-4)$ & 0.7 \\
\hline \multicolumn{4}{|l|}{ Caregiver Burden Scale } \\
\hline General strain & $2.63(1.88-3.0)$ & $1.88(1.75-2.56)$ & 0.02 \\
\hline Social isolation & $2.33(1.48-3.33)$ & $2.33(1.33-2.75)$ & 0.037 \\
\hline Disappointment & $2.6(1.8-3.00)$ & $2.4(1.8-3.0)$ & 0.65 \\
\hline Emotional involvement & $1.33(1.22-2.33)$ & $1.33(1.25-2.0)$ & 0.77 \\
\hline Environment & $1.67(1.22-2.22)$ & $1.67(1.0-2.0)$ & 0.29 \\
\hline Total burden index & $2.41(1.76-2.77)$ & $2.0(1.58-2.42)$ & 0.12 \\
\hline \multicolumn{4}{|l|}{ General Health Questionnaire } \\
\hline Total & $3(0-7)$ & $1(0-2.25)$ & 0.05 \\
\hline
\end{tabular}


correlations with BSSS: perceived available emotional support $(r=-0.6, p=0.001)$, perceived available instrumental support $(r=-0.42, p=0.005)$, actually received informational support $(r=-0.5, \quad p=0.01)$, and instrumental support $(r=-0.55, p=0.001)$. GHQ-12 correlated negatively with perceived available emotional support $(r=-0.49, p=0.001)$, perceived available instrumental $\operatorname{support}(r=-0.33, p=0.03)$, actually received informational $(r=-0.38, p=0.02)$, and instrumental support $(r=-0.46, p=0.003)$.

In the group of secondary caregivers, no significant correlation was found between PedsQL test scores (total score) and GHQ-12, CBS, and BSSS results, with the exception of one subscale: support seeking $(r=0.66, p=0.004)$. Significant positive correlations were demonstrated between GHQ-12 and total burden index in CBS $(r=0.71, p=001)$ and negative correlations between GHQ-12 and the BSSS subscales perceived available support (emotional: $r=-0.54, p=0.03$, instrumental: $r=-0.57$, $p=0.02$ ), and actually received support (emotional: $r=-0.54$, $p=0.03$, informational: $r=-0.5, \quad p=0.04, \quad$ instrumental: $r=-0.69, p=0.002)$. The CBS total burden index correlated negatively with the BSSS subscales perceived available support (emotional: $r=-0.53, p=0.03$, instrumental: $r=-0.48$, $p=0.05$ ), and actually received support (emotional: $r=-0.49, p=0.05$ and instrumental: $r=-0.66, p=0.004$ ).

\section{Discussion}

This paper presents for the first time the psychosocial aspects in families of Polish children with ESRD treated with APD. The studied group, although small, can be regarded as representative of the Polish population of children aged $\geq 2$ treated with APD. Since 2003, a decline in the number of children treated with dialysis has been observed due to the increased number of kidney transplants [18]. Furthermore, children $\leq 2$ years of age in Poland constitute a significant proportion of patients on PD who, along with their parents, are not included in the study due to the established evaluation criteria. It would, therefore, seem that the obtained data may be considered not accidental for the illness case studied by us.

The few studies on the psychosocial situation of children treated with PD and their families published thus far have dealt with small groups of patients, inter alia, 32 patients and their 32 guardians [19], four families (14 people in all) [20], 17 patients and ten parents [21], 20 children and 28 parents [4]. Some studies focused on children treated with dialysis regardless of type [22-24]. However, the dialysis method is known to be one of the important factors influencing the QoL of adults with CKD and their families [25, 26].

Our survey data show a harsh economic situation in the studied families. However poor the financial situation is in families of children on ADP, no definite conclusion can be drawn that it is the result of the child's illness. Data on parents' occupations and sources of income before the child's illness were not collected. It must be emphasized that $40 \%$ of mothers and $45.71 \%$ of fathers claimed the deterioration of financial situation followed the diagnosis of their child's illness.

Almost half of the examined families receive social security benefits that, in Poland, are awarded to families with very low income. Nearly $75 \%$ of mothers do not work, which may be explained by the need to look after a child with ESRD. As demonstrated in the studied group, mothers also act as caregivers in $98 \%$ of the cases. It was also observed that nearly $20 \%$ of fathers do not work. Improvement in families' economic situations seems to be the "field of action" for social workers. This area, however, reveals certain inconsistencies: $42 \%$ of mothers have never dealt with social workers and another $10 \%$ evaluated their interactions with said workers as not good $(56 \%$ and $20 \%$ of fathers, respectively). A poor contact with social workers in parents' valuation/opinion may stem from the fact that access to social workers and psychologists is on an ad hoc basis. These people probably do not know the dialysis treatment specificity. Our findings regarding the socioeconomic situation (SES) of families of PD patients are consistent with those of other authors who recognize SES as one of the important factors that determine the quality of care for PD patients $[19,27]$.

The positive observation is that nearly all children attend schools with standard education programs. As other authors have pointed out, education is an important factor in preparing children for participation in adult life [28]. It should be noted, however, that $>50 \%$ of children take advantage of individual tutoring. It may improve efficiency but on the other hand may lead to social isolation and limited interpersonal communication. More than $60 \%$ of $\mathrm{PD}$ patients require more help with schoolwork, which is provided by parents and which may constitute an additional burden.

A child's illness affects changes in the family that most often include living space rearrangement, participation of additional individuals in caring for the sick child, and the deterioration of existing social relationships. Parents often only establish new friendships with other sick children's parents. This is beneficial in exchanging experiences; however, it also indicates the affected families' isolation. Any information provided by the parents about changes in the family connected with the child's disease should be taken into consideration during contacts with medical personnel. They should be mentioned in conversations. It may help members of the family adapt to this dialysis situation.

Our study shows positive relationships between parents and medical personnel in parents' opinions. Expectations toward medical personnel included more information regarding the child's illness. It is alarming that $>63 \%$ of primary caregivers asserted that kidney transplantation had not been discussed with 
them. This seems inconceivable considering the standards that exist in Poland; however, it does suggest that information regarding this issue is insufficient, was not relayed in a comprehensible manner, or the parents were experiencing intellectual difficulties understanding it.

The perceived HRQoL among the surveyed children was low, which is consistent with the findings of most authors who have used the PedsQL test $[22,29]$. HRQoL is a very individualized, subjective perception based on feelings, but different scales are based only on observable indicators of the health condition. Patients rated their school and physical functioning as particularly low. It is worth mentioning that the overall QoL as well as physical and emotional functioning was rated decidedly lower by parents than by their children. Similar results were obtained by other authors who dealt with chronically sick children of different etiology [6]. This situation may lead to overprotectiveness and thus hamper the child's development of self-reliance and selfsufficiency and, as a result, amplify the perceived burden experienced by the parents. This hypothesis is supported by the observed correlations between test scores among parents.

Our study shows that parents/primary caregivers evaluated the level of overall burden as medium. Contrary to expectations, a high level of burden pertained to a relatively small proportion of caregivers. It can be assumed that acting as a caregiver also carries positive effects that reduce the perceived burden [30]. Secondary caregivers rated the burden as low or medium. This observation is consistent with the claimed improvement of relationships with the sick child by the parents (or lack of changes toward the child) and the evaluation of the relationship with the patient as very good in most cases. In turn, high levels of the perceived burden were observed in the following areas: physical and mental fatigue, responsibility for the child's well-being, extended time devoted to the child and lack of time for oneself, being limited by the child's affairs, and disregard for one's own health. Parents also obtained high scores on the disappointment scale. In their assessment, conditions such as financial sacrifices connected with the illness, inability to fulfil own plans, loneliness, and isolation were common. Studies have not, however, suggest feelings such as pervasive sadness, resentment, guilt, or loss to be present among caregivers of adult patients on dialysis [31].

In order to assess parents' needs, we applied several scales, including BSSS. Social support, as defined by Schwarzer and Schulz, includes resources and help provided by other people [32]. Researchers and theorists distinguish different types of social support: instrumental support (sharing certain goods), informational support (advice on how to solve a problem), and emotional (comforting and showing compassion) [33]. Our research findings suggest that sick children's parents have the need for social support. Comparing said findings with observations by other authors shows that the need for support is higher in those parents than in parents of healthy individuals, as well as in patients with chronic conditions such as cardiovascular and musculoskeletal diseases [16]. Research by Duits et al. suggests that the reported need for support may be a predictor of depression or anxiety [34]. At the same time, parents identify the possibility of such support and report seeking support, i.e., other people's help. These observations evidence parents' adaptation to their child's illness, which may stem from its relatively long duration. However, it should be noted that the received support is lower than the need for it. According to the Schwarzer and Leppin theory, received support is a factor in reducing risk in stress situations [35]. In this context, the obtained results indicate the occurrence of insecurity, anxiety, and depression among parents. Our study shows that parents most often receive instrumental support while mostly lacking emotional support.

The GHQ-12 is a screening instrument aimed at assessing the mental condition of adults in the general population. It allows us to assess the increase of nonpsychotic mental disorders and to select people who are most likely to develop them. The feeling of mental disorder may be also assessed with GHQ - as well as the subjective assessment of its results - called nonspecific psychological distress. Over half of primary caregivers received GHQ12 scores indicating the possible occurrence of abnormal mental functioning. No mental history of parents was obtained, so it is impossible to unambiguously indicate the basis for such mental disorders. However, the results may signal anxiety, depression, and the loss of confidence as a reaction to difficulties and the inability to fulfill their own goals and objectives. We found correlations between assessment of the parent's own mental health and perceived available support (emotional, informational, and instrumental), which suggests that external environmental factors may be an important determinant of an individual's mental state. The results underline the strong need to provide continuous psychological care for caregivers.

Among parents, most tests demonstrated no significant differences between primary caregivers and secondary parents. The former indicated, however, significantly higher general strain and poorer overall mental health as a consequence of greater burdens. Our study revealed no significant pattern in the dimensions of psychosocial functioning among parents based on parameters such as age, education, professional career, family structure (complete-single parent), having or not having other children, and source of income (professional career vs. welfare benefits). The demonstrated higher overall burden among women stems from their role as primary caregivers. Interesting observations were made with regards to the influence of place of residence on psychosocial functioning. Residents in medium-sized cities are at a disadvantage in terms of the sense of social isolation, overall burden, and perceived available instrumental support. This may stem from anonymity and loosening of social ties, more intensified need (as opposed to villages), or lack of access to take advantage of various benefits of modern, urban life easily available in large cities. 
Our study has its limitations. The research material, though representative, is not extensive, and includes children of different ages. The research did not include families of children $<2$ years of age, as caring for them may be more challenging and the shorter duration of the situation and lack of adaptation to it may be a source of many problems. It would be necessary to conduct prospective studies in order to analyze the dynamics of changes in the process of adapting to the burdens related to performing dialysis. The QoL in patients' families as a multidimensional concept requires the use of multidimensional tools in its assessment. Thus far, no appropriate tools have been developed to evaluate the psychosocial situation of families of patients with CKD. Results may, therefore, prove difficult to compare between studies, as there may be little or no similarities between the tools used. Nevertheless, we believe that our findings may prove useful in determining the direction of future studies of psychosocial help provided to children on APD and their families, as well as formulating initial support programs in this area. We were largely unable to carry out tests of both parents. Lack of response from fathers points to the polarization of roles in the families of sick children. Observations of similar perceptions pertaining to changes in the family after the diagnosis, relationships with individuals in the immediate surroundings, burdens, and social support regardless of the degree of involvement in caring for a sick child are all areas that require further research. The findings may suggest ways to help support harmony in families and also different ways of helping families adapt to having a member with CKD.

In conclusion, this study shows that the financial situation in families of children treated with APD is challenging. It is necessary to expand the involvement of social workers in helping patients' families. Despite rating the level of overall burden as medium, parents who provide the primary care to children on PD report limitations in self-realization and depend upon emotional support. More than half of them may have impaired mental function. There is the strong need to provide continuous psychosocial care for caregivers. Contrasting perceptions of children's overall QoL and physical and emotional functioning, as experienced by the children themselves and their primary caregivers, may result in challenges in the patient's adaptation to adult life. Determinants of the PD patient's family's ability to cope with the burden of care require further research.

Open Access This article is distributed under the terms of the Creative Commons Attribution License which permits any use, distribution, and reproduction in any medium, provided the original author(s) and the source are credited.

\section{References}

1. Schaefer F, Warady BA (2011) Peritoneal dialysis in children with end-stage renal disease. Nat Rev Nephrol 7:659-668
2. Zaritsky J, Warady BA (2011) Peritoneal dialysis in infants and young children. Semin Nephrol 31:213-224

3. Lai WM (2009) Quality of life in children with end-stage renal disease: does treatment modality matter? Perit Dial Int Suppl 2: S190-S191

4. Tong A, Lowe A, Sainsbury P, Craig JC (2008) Experiences of parents who have children with chronic kidney disease: a systematic review of qualitative studies. Pediatrics 121:349-360

5. de Paula ES, Nascimento LC, Rocha SM (2008) Roles assessment in families of children with chronic renal failure on peritoneal dialysis. Int J Nurs Pract 14:215-220

6. Morrow AM, Hayen A, Quine S, Scheinberg A, Craig JC (2012) A comparison of doctors', parents' and children's reports of health states and health-related quality of life in children with chronic conditions. Child Care Health Dev 38:186-195

7. Tong A, Lowe A, Sainsbury P, Craig JC (2010) Parental perspectives on caring for a child with chronic kidney disease: an in-depth interview study. Child Care Health Dev 36:549-557

8. Abdel-Kader K, Myaskovsky L, Karpov I, Shah J, Hess R, Dew MA, Unruh M (2009) Individual quality of life in chronic kidney disease: influence of age and dialysis modality. Clin J Am Soc Nephrol 4:711-718

9. Varni JW, Burwinkle TM, Katz ER (2003) The PedsQL 4.0 as a pediatric population health measure: feasibility, reliability, and validity. Ambul Pediatr 3:329-341

10. Varni JW. PedsQL TM (Pediatric Quality of Life Inventory TM). www.pedsql.org

11. Goldberg D, The General Health Questionnaire. http://www. gl-assessment.co.uk/products/general-health-questionnaire-0

12. Schulz U, Schwarzer R (2003) Social support in coping with illness: the Berlin Social Support Scales (BSSS). Diagnostica 49:73-82

13. Schwarzer R. Berlin Social-Support Scales (BSSS). http:// userpage.fu-berlin.de/ health/soc pol.htm, 5.10.2011

14. Elmstahl S, Malmberg B, Annerstendt L (1996) Caregiver's burden of patients 3 years after stroke assessed by a novel caregiver burden scale. Arch Phys Med Rehabil 77:177-182

15. Makowska Z, Merecz D (2001) Mental health assessment on a research basis by David Goldberg Questionnaires. Nofer Institute of Occupational Medicin, Łódź [in Polish]

16. Łuszczyńska A, Smith M, Mazurkiewicz M, Schwarzer R (2006) Berlin Social Support Scales (BSSS). The results of initial research on the adaptation of the scales and their psychometric properties. Psychol Stud 44:17-27

17. Jaracz K, Grabowska-Fudala B (2007) The caregiver's burden scale. Polish version. Publishing House of Poznan University of Medical Sciences, Poland

18. Grenda R, Rubik J, Rutkowski B (2010) Report on renal replacement therapy in children in Poland 2010. Polish Ministry of Health, Warszawa

19. Tsai TC, Liu SI, Tsai JD, Chou LH (2006) Psychosocial effects on caregivers for children on chronic peritoneal dialysis. Kidney Int 70:1983-1987

20. de Paula ES, Nascimento LC, Rocha SM (2008) The influence of social support on strengthening families of children with chronic renal failure. Rev Lat Am Enfermagem 16:692-699

21. Chiu MC, Ng CF, Lee LP, Lai WM, Lau SC (2007) Automated peritoneal dialysis in children and adolescents - benefits: a survey of patients and parents on health-related quality of life. Perit Dial Int 27(Suppl 2):S138-S142

22. McKenna AM, Keating LE, Vigneux A, Stevens S, Williams A, Geary DF (2006) Quality of life in children with chronic kidney disease-patient and caregiver assessments. Nephrol Dial Transplant 21:1899-1905

23. Wiedebusch S, Konrad M, Foppe H, Reichwald-Klugger E, Schaefer F, Schreiber V, Muthny FA (2010) Health-related quality 
of life, psychosocial strains, and coping in parents of children with chronic renal failure. Pediatr Nephrol 25:1477-1485

24. Buyan N, Türkmen MA, Bilge I, Baskin E, Haberal M, Bilginer Y, Mir S, Emre S, Akman S, Ozkaya O, Fidan K, Alpay H, Kavukcu S, Sever L, Ozçakar ZB, Dogrucan N (2010) Quality of life in children with chronic kidney disease (with child and parent assessments). Pediatr Nephrol 25:1487-1496

25. Morton RL, Tong A, Webster AC, Snelling P, Howard K (2011) Characteristics of dialysis important to patients and family caregivers: a mixed methods approach. Nephrol Dial Transplant 26:4038-4046

26. Boateng EA, East L (2011) The impact of dialysis modality on quality of life: a systematic review. J Ren Care 37:190-200

27. Bruce MA, Beech BM, Sims M, Brown TN, Wyatt SB, Taylor HA, Williams DR, Crook E (2009) Social environmental stressors, psychological factors, and kidney disease. J Investig Med 57:583-589

28. Groothoff JW, Grootenhuis MA, Offringa M, Stronks K, Hutten GJ, Heymans HS (2005) Social consequences in adult life of endstage renal disease in childhood. J Pediatr 146:512-517
29. Goldstein SL, Graham N, Burwinkle T, Warady B, Farrah R, Varni JW (2006) Health-related quality of life in pediatric patients with ESRD. Pediatr Nephrol 21:846-850

30. Andrén S, Elmståhl S (2005) Family caregivers' subjective experiences of satisfaction in dementia care: aspects of burden, subjective health and sense of coherence. Scand J Caring Sci 19:157-168

31. White Y, Grenyer BF (1999) The biopsychosocial impact of end-stage renal disease: the experience of dialysis patients and their partners. J Adv Nurs 30:1312-1320

32. Schulz U, Schwarzer R (2004) Long-term effects of spousal support on coping with cancer after surgery. J Soc Clin Psychol 23:716-732

33. Pierce GR, Sarason IG, Sarason BR (1991) General and relationship-based perceptions of social support: are two constructs better than one? J Pers Soc Psychol 61:1028-1039

34. Duits AA, Boeke S, Taams MA, Passchier J, Erdman RA (1997) Prediction of quality of life after coronary artery bypass graft surgery: a review and evaluation of multiple, recent studies. Psychosom Med 59:257-268

35. Schwarzer R, Leppin A (1991) Social support and health: a theoretical and empirical overview. J Soc Pers Relat 8:99-127 